\title{
Proposal for Inverse-Phase Composite Zone Plate for Deeper Depth of Focus
}

\author{
Yasushi Kagoshima* and Yuki Takayama
}

Graduate School of Material Science, University of Hyogo, 3-2-1 Kouto, Kamigori, Ako, Hyogo 6781297, Japan

*Corresponding author: kagosima@sci.u-hyogo.ac.jp

Zone plates (ZPs), including their derivations, and total reflection mirrors have facilitated the realization of sub-100-nm spatial resolution, and the demand for localized analysis of practical samples using X-ray microscopy is rapidly increasing. According to the optical principle in a focusing optical system, the smaller the focused beam size is, the shallower is the depth of focus (DoF). Usually, diffraction limit in optics means that the product of a spatial resolution $\left(\Delta_{\text {res. }}\right)$ and the numerical aperture (NA) cannot be smaller than the wavelength, i.e., $\Delta_{\text {res. }} \times \mathrm{NA} \geq \lambda$. Because DoF is also determined by NA (DoF $\propto 1 / \mathrm{NA}^{2}$ ), smaller $\Delta_{\text {res. }}$ and deeper DoF cannot be simultaneously attained, which is another viewpoint of the diffraction limit. This limit restricts the analysis of thicker samples than DoF and is a bottleneck in highspatial-resolution observation of practical samples using X-ray microscopy.

We attempt to relax this limit using a wave-optical approach. In this paper, a novel type of ZP, called inverse-phase composite ZP (IP-CZP), is proposed and examined to make the DoF deeper with little vitiation in the spatial resolution. We must mention that a fractal ZP was also proposed for deep focus [1].

ZPs are classified into two types: positive and negative ZPs, as shown in Figs. 1(a) and 1(b), respectively. Both ZPs function as a thin lens. They have the same focal intensity distribution both in the focal plane (which determines $\Delta_{\text {res. }}$ ) and along the optical axis (which determines DoF), but the phase of the complex amplitude is opposite to each other. A composite ZP was proposed, and its optical properties were well studied [2]. If the inner ZP (iZP) with first-order focal length $f$ is surrounded by the outer ZP (oZP), also with third-order focal length $f$, the effective NA becomes larger, as shown in Fig. 1(c), which leads to a gain in both the spatial resolution and focused intensity.

To make the DoF deeper, the peak of the focal intensity distribution along the optical axis should be suppressed, whereas the focusing beam shape in the focal plane should be kept constant as much as possible. The strategy to accomplish this is as follows. We propose an idea that considers the case where the phase of iZP and oZP is inversely composed, as shown in Fig. 1(d). Because the aperture of oZP is annular, the width of the focusing beam becomes narrower than that of a circular aperture with the same NA. If $3 N_{\text {out_eff }}$ is sufficiently smaller than $N_{\text {in }}$ ( $N_{\text {out_eff: }}$ effective number of zones of oZP; $N_{\text {in: }}$ number of zones of iZP; 3 denotes a third-order focus), the monochoromaticity requirement for oZP becomes relax,

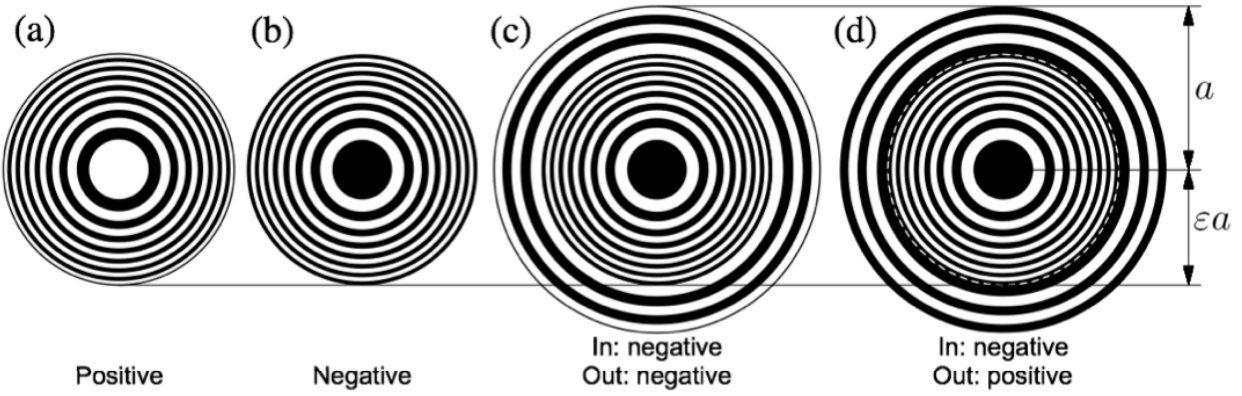

Figure 1. (a) Positive ZP, (b) negative ZP, (c) composite ZP, and (d) inverse-phase composite ZP. 
which leads to the broadening in the focal intensity distribution of oZP along the optical axis. Therefore, if the parameters of the IP-CZP are appropriately chosen, the DoF can be deepened with little vitiation in the spatial resolution.

The main variable design parameters are minimum fabrication width $\Delta r_{\text {fab }}$, thickness ratio $\eta$ (ratio of the oZP zone thickness to the iZP one), annular aperture parameter $\varepsilon$ (oZP radius $a$ and iZP radius $\varepsilon a$ ), and initial phase of oZP $\zeta$. Diffraction integration has been performed to calculate the two-dimensional complex amplitude distribution (magnitude, phase, and real and imaginary parts) near the focal point. One dimension is the radial direction, and the other dimension is the optical-axis direction. The main conditions are chosen as follows: $\mathrm{X}$-ray energy is $10 \mathrm{keV}$, zone material is tantalum, the outermost zone width of iZP, that is, $\Delta r_{N}$ in, is $84 \mathrm{~nm}$, and the innermost radii of iZP, namely, $r_{1}$ in , and that of oZP, namely, $r_{1 \text { ou }}$, are $2.50 \mu \mathrm{m}$ and $(3)^{1 / 2} r_{1}$ in $\mu \mathrm{m}$, respectively, which result in focal length $f$ of $50.41 \mathrm{~mm}$. The outer radii of iZP and oZP are denoted as $r_{N_{-} \text {in }}$ and $r_{N_{-} \text {out }}$, respectively. The boundary radius is denoted as $r_{N_{\text {in }}}$ in which the zone width of iZP, that is, $\Delta r_{N}$ in, is equal to $\Delta r_{\text {fab }}$. As an initial design step, the zone thickness of iZP, namely, $t_{\text {in }}$, is chosen to be $2.645 \mu \mathrm{m}$, yielding a phase shift of $\pi$, which means that the iZP works as an ordinary phase ZP. The thickness of oZP is denoted as $\eta t_{\text {in }}$.

Figures 2(a)-2(c) show the calculated two-dimensional intensity distribution near the focal point, the point spread function (PSF), and intensity distribution along the optical axis, respectively, in the case of $\varepsilon=0.84$, $\eta=0.85$, and $\zeta=0$. The red and blue lines in Figs. 2(b) and 2(c) correspond to the IP-CZP and iZP only. The black dotted lines in Fig. 2(a) denote the half value. Radius $r_{\text {null, }}$ which initially yields zero, is 109.3 $\mu \mathrm{m}$, and the DoF in the IP-CZP is $412.3 \mu \mathrm{m}$. Because $\Delta_{\text {res. }}$ determined by $\Delta r_{\text {fab }}$ is $102.5 \mu \mathrm{m}\left(=1.22 \Delta r_{\text {fab }}\right)$, the vitiation in the spatial resolution is as small as $7 \%$. On the other hand, the DoF can be 1.8 times deeper than that determined by $\Delta r_{\text {fab }}$ of $227.8 \mu \mathrm{m}\left(=4 \Delta r_{\mathrm{fab}}{ }^{2} / \lambda\right)$. Further, a flat-intensity focus along the optical axis is also attained. We found that a DoF of nearly twice the depth could be expected with little vitiation in the spatial resolution.

\section{References}

[1] G Saavedra et al., Opt. Lett. 28 (2003) 971.

[2] A G Michette, Optical Systems for Soft X-Rays (Plenum, New York, 1986).

This work was supported by JSPS KAKENHI Grant Number JP16K05019. We would like to thank Editage (www.editage.jp) for the English language editing.
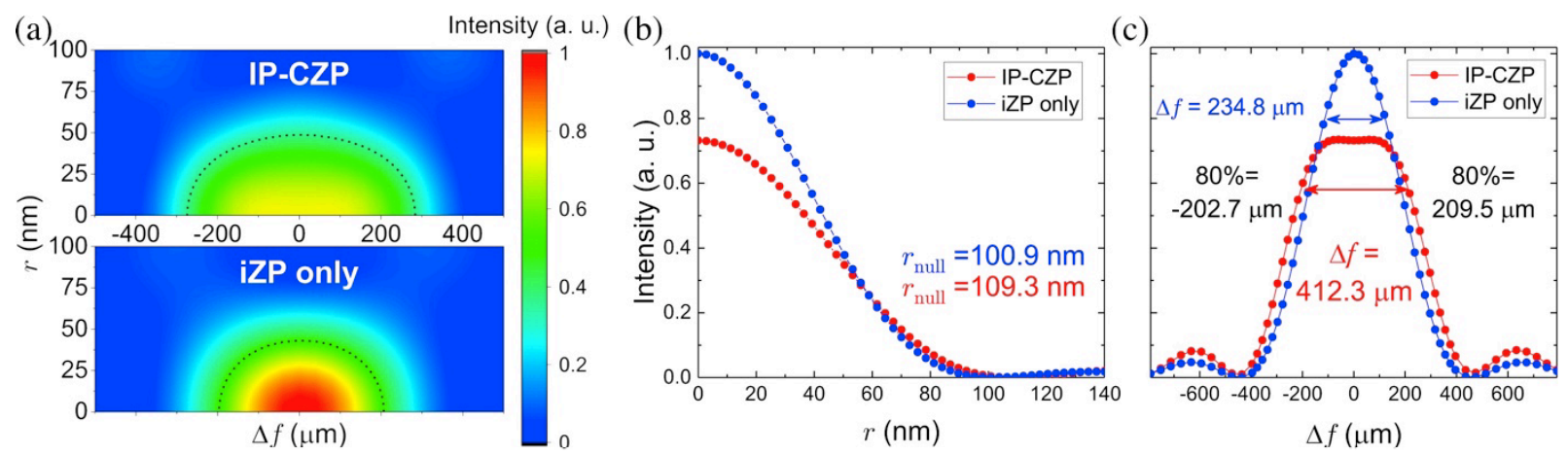

Figure 2. (a) Calculated two-dimensional intensity distribution of IP-CZP and only iZP near the focal point. (b) PSFs and (c) intensity changes along the optical axis. 